\title{
Retraction Note: TLE3 represses colorectal cancer proliferation by inhibiting MAPK and AKT signaling pathways
}

\author{
Run-Wei Yang ${ }^{1,2,3 \dagger}$, Ying-Yue Zeng ${ }^{1,2,3+}$, Wen-Ting Wei ${ }^{1,2,3 \dagger}$, Yan-Mei Cui ${ }^{1,2,3}$, Hui-Ying Sun ${ }^{1,2,3}$, Yue-Long Cai ${ }^{1,2,3}$, \\ Xin-Xin Nian ${ }^{1,2,3}$, Yun-Teng Hu 1,2,3 $^{1,3 u-P i n g}$ Quan ${ }^{1,2,3}$, Sheng-Lu Jiang ${ }^{1,2,3}$, Meng Wang ${ }^{1,2,3}$, Ya-Li Zhao ${ }^{1,2,3}$, \\ Jun-Feng Qiu ${ }^{1,2,3}$, Ming-Xuan Li ${ }^{1,2,3}$, Jia-Huan Zhang ${ }^{1,2,3}$, Mei-Rong He ${ }^{4}$, Li Liang ${ }^{1,2,3}$, Yan-Qing Ding ${ }^{1,2,3,5^{*}}$ and \\ Wen-Ting Liao ${ }^{1,2,3,5^{*}}$
}

\section{Retraction Note: J Exp Clin Cancer Res 35, 152 (2016) https://doi.org/10.1186/s13046-016-0426-8}

The Editor-in-Chief has retracted this article at the request of Wen-Ting Liao. The author stated that the data presented in Fig. 2F (HE and Ki67) were published in the authors' previous study [1], and the data in Figs. 1C $(\alpha$-tubulin, FOXO3, p-AKT) and 2A ( $\alpha$-tubulin) were published in [2]. In addition, the same $\alpha$-tubulin western blot bands are presented in Figs. 2A and 3A. Thus, the corresponding author stated that the authors have no confidence in the reliability of the data and conclusions reported in this article. Run-Wei Yang, Ying-Yue Zeng, Wen-Ting Wei, Yan-Mei Cui, Hui-Ying Sun, Li Liang, and Wen-Ting Liao agree to this retraction. Yue-Long Cai, Xin-Xin Nian, Yun-Teng $\mathrm{Hu}, \mathrm{Yu}$-Ping Quan, Sheng-Lu Jiang, Meng Wang, Ya-Li Zhao, Jun-Feng Qiu, Ming-Xuan Li, Jia-Huan Zhang, Mei-Rong He, and YanQing Ding have not responded to any correspondence about this retraction.

\section{Author details}

'Department of Pathology, Nanfang Hospital, Southern Medical University, Guangzhou 510515, Guangdong, China. ${ }^{2}$ Department of Pathology, School of Basic Medical Sciences, Southern Medical University, Guangzhou,

Guangdong, China. ${ }^{3}$ Guangdong Provincial Key Laboratory of Molecular

The original article can be found online at https://doi.org/10.1186/s13046 016-0426-8.

* Correspondence: dyq@fimmu.com; liaowt2002@gmail.com

${ }^{\dagger}$ Run-Wei Yang, Ying-Yue Zeng and Wen-Ting Wei contributed equally to this work.

'Department of Pathology, Nanfang Hospital, Southern Medical University, Guangzhou 510515, Guangdong, China

Full list of author information is available at the end of the article
Tumor Pathology, Guangzhou, Guangdong, China. ${ }^{4}$ Guangdong Provincial Key Laboratory of Gastroenterology, Department of Gastroenterology, Nanfang Hospital, Southern Medical University, Guangzhou, Guangdong, China. ${ }^{5}$ Department of Pathology, Nanfang Hospital and School of Basic Medical Sciences, Southern Medical University, Guangzhou 510515,

Guangdong, China.

Published online: 01 September 2021

References

1. Cui Y-M, Jiang D, Zhang S-H, Wu P, Ye YP, Chen CM, et al. FOXC2 promotes colorectal cancer proliferation through inhibition of FOXO3a and activation of MAPK and AKT signaling pathways. Cancer Lett. 2014;353(1):87-94. https://doi.org/10.1016/j.canlet.2014.07.008.

2. Cui YM, Jiao HL, Ye YP, Chen CM, Wang JX, Tang N, et al. FOXC2 promotes colorectal cancer metastasis by directly targeting MET. Oncogene. 2015; 34(33):4379-90. https://doi.org/10.1038/onc.2014.368.

C C The Author(s). 2021 Open Access This article is licensed under a Creative Commons Attribution 4.0 International License, which permits use, sharing, adaptation, distribution and reproduction in any medium or format, as long as you give appropriate credit to the original author(s) and the source, provide a link to the Creative Commons licence, and indicate if changes were made. The images or other third party material in this article are included in the article's Creative Commons licence, unless indicated otherwise in a credit line to the material. If material is not included in the article's Creative Commons licence and your intended use is not permitted by statutory regulation or exceeds the permitted use, you will need to obtain permission directly from the copyright holder. To view a copy of this licence, visit http://creativecommons.org/licenses/by/4.0/ The Creative Commons Public Domain Dedication waiver (http://creativecommons.org/publicdomain/zero/1.0/) applies to the data made available in this article, unless otherwise stated in a credit line to the data. 